MECHANICAL VENTILATION

\title{
Outcomes, cost and long term survival of patients referred to a regional weaning centre
}

\author{
D V Pilcher, M J Bailey, D F Treacher, S Hamid, A J Williams, A C Davidson
}

Thorax 2005;60:187-192. doi: 10.1136/thx.2004.026500

See end of article for authors' affiliations

Correspondence to: Dr A C Davidson, Lane Fox Respiratory Unit, Guy's and St Thomas' Hospital, London SEI 7EH, UK; craig.davidson@gstt. sthames.nhs.uk

Received 10 April 2004 Accepted 17 October 2004

\begin{abstract}
Background: Regional weaning centres provide cost effective care for patients who have undergone prolonged mechanical ventilation. There are few published European data on outcomes in these patients. Methods: Patients admitted for weaning to the Lane Fox Respiratory Unit (LFU) between January 1997 and December 2000 were identified. The proportion weaned from mechanical ventilation, in-hospital mortality, and subsequent survival after discharge were examined.

Results: A total of 153 patients had been ventilated for a median of 26 days before transfer. The daily cost per patient stay was $€ 1350$. Fifty eight patients $(38 \%)$ were fully weaned, $42(27 \%)$ died, and $53(35 \%)$ required ventilatory support at discharge from hospital of whom $36(24 \%)$ required only nocturnal ventilation. Univariate analysis showed increasing age (OR 1.06, $<<0.001$ ), length of ICU stay (OR 1.02, $p=0.001)$, APACHE II predicted risk of death score (OR 1.02, $p=0.05)$, and a surgical cause for admission (OR 4.04) were associated with mortality. Neuromuscular/chest wall conditions were associated with low mortality (OR 0.36 ) but low likelihood of weaning from ventilation (OR 0.28 ). Female sex (OR 2.13, $p=0.03$ ) and COPD (OR 2.81) were associated with successful weaning. Overall survival at 3 years from admission was $47 \%$. Long term survival was lowest in patients with COPD.

Conclusions: Most patients survived to leave hospital, the majority having been liberated from ventilatory support. Survivors were younger and spent less time ventilated in the referring ICU. The underlying diagnosis determined success of weaning, hospital survival, and long term outcome.
\end{abstract}

$M$ ost patients who require mechanical ventilation during critical illness can be successfully liberated from respiratory support on recovery from the precipitating illness. For $2-5 \%$ of patients, however, the weaning process fails, conventionally defined as dependency on mechanical ventilation more than 3 weeks after resolution of the precipitating cause for admission to the intensive care unit (ICU). Specialist weaning centres, geographically distant from general ICUs, have been developed, mostly in the USA, to manage such weaning failure patients. Variable mortality and weaning success has been reported. ${ }^{1-12}$

The Lane Fox Unit (LFU) is a 16-bed facility, staffed with a nurse to patient ratio of $3.5: 1$ and equipped to provide invasive (tracheostomy) and non-invasive (NIV) support. Before 1995, experience was largely in caring for chronic ventilator dependent patients in the community. A service was developed recognising the needs of a larger group of patients in general ICUs with weaning delay. A policy of transfer of responsibility of care and long term placement of those failing to wean was adopted. Exceptionally, patients considered unable to be weaned were transferred back to the referring institution after initial evaluation.

The unit provides all forms of ventilatory support including negative pressure tanks, cuirass jackets, rocking beds, and nose/face mask positive pressure techniques. Extensive use of NIV as a bridge, particularly in sleep, to spontaneous breathing underlies the approach to weaning. This is coupled with techniques to clear respiratory secretions (breath stacking, frog breathing, and insufflation/exsufflation). The weaning process involves daily targets of either increasing periods of T-piece ventilation and/or a gradual reduction in pressure support. Assist control ventilation was provided during sleep. Other aspects include identification of reversible causes of weaning failure (such as unnecessary steroids), avoidance of sedatives, restoration of normal sleep/ wake cycles, attention to nutrition, proactive physiotherapy including ambulatory ventilation, and attempts to improve patient autonomy such as establishing speech.

This is a report of our experience over a 4 year period. We have examined the influence of the underlying diagnosis on the success of weaning and outcome, and also report on costs and long term survival which have only rarely been reported previously.

\section{METHODS}

The study was approved by the ethics committee of Guy's and St Thomas' Hospital. All patients transferred to the LFU between January 1997 and December 2000 were identified and included if referred because of evident or expected failure to wean from mechanical ventilation. The clinical notes, nursing charts, and computer databases were inspected and, when not otherwise available, additional information was sought from the referring hospital by letter and telephone. Patients transferred to other hospitals from the LFU were traced. Long term survival data were obtained by contacting the patients' general practitioners, referring hospitals, and by using death certificate data generated by the Office of National Statistics for England and Wales.

Reimbursement from the referring hospital or health authority was calculated from the Therapeutic Intervention Scoring System (TISS) using a method previously described for intensive care patients. ${ }^{13}$ A fixed cost was calculated for each patient which reflected medical, technician and clerical salary costs as well as capital equipment depreciation, equipment and capital hospital costs. A variable cost was measured for each patient on a daily basis. This included nursing, physiotherapy, radiology, and pathology staff costs and consumables. Patients were scored until weaned from invasive ventilation or discharge from LFU, whichever came first.

Age, sex, principal underlying diagnosis, time ventilated in the referring ICU, duration of stay in LFU, APACHE II score 
on admission to the referring ICU, and cost of stay in LFU were recorded. Causes of respiratory failure leading to prolonged mechanical ventilation were classified into four diagnostic groups: neuromuscular/chest wall disorders; chronic obstructive pulmonary disease (COPD); surgical; and other diagnoses (which included conditions such as non-COPD respiratory disease, obesity hypoventilation, left ventricular failure, and central nervous system disorders).

Primary outcome measures were ventilator independence, hospital mortality, and long term mortality. Weaning success was defined as being free from any form of ventilatory assistance at discharge from hospital. Any requirement for ventilation during the day was classified as "full ventilation" as opposed to those who only required nocturnal ventilation. Patients who weaned from ventilation but died before leaving hospital were included in the deaths group rather than in the "weaned from ventilation" group.

\section{Statistical analysis}

All data were analysed using SAS Version 8.0 (SAS Institute Inc, Cary, NC, USA). Non-parametric data were expressed as median and interquartile range and a $\mathrm{p}$ value of $<0.05$ was considered to be statistically significant. Univariate analysis was performed using $t$ tests, logistic regression, $\chi^{2}$ tests for equal proportion, Spearman correlation coefficients, Wilcoxon rank sum tests, and log rank tests, where appropriate. Multivariate analysis was performed on survival time using Cox proportional hazards regression models. Kaplan-Meier survival curves are shown for the whole group and for the three largest diagnostic groups.

\section{RESULTS}

Between the beginning of January 1997 and end of December 2000, 153 patients (who had 157 admissions) were identified as weaning referrals to the LFU. One patient had three separate admissions and two further patients had two admissions each. These were all separate hospitalisation episodes. Over the study period nine patients were referred but not transferred. Four had associated neurological pathology that made weaning very unlikely, three weaned successfully while on the waiting list, and two died.

Demographic data of the group are shown in table 1. The three largest diagnostic groups were neuromuscular/chest wall disorders $(31 \%, \mathrm{n}=47), \mathrm{COPD}(27 \%, \mathrm{n}=41)$, and surgical $(24 \%, \mathrm{n}=36)$. The surgical group consisted of 13 patients who had undergone cardiac surgery, seven gastrointestinal surgery, three major vascular surgery, three trauma, and 10 others. The neuromuscular/chest wall group comprised patients with chest wall disorders, neuropathies and myopathies (nine idiopathic kyphoscoliosis, eight postpolio syndrome, eight motor neurone disease, six GuillainBarré, four Duchenne's muscular dystrophy, four myotonic

\begin{tabular}{ll|}
\hline $\begin{array}{l}\text { Table } 1 \\
\text { ( } \mathrm{n}=153)\end{array}$ & Demographic data of study group \\
\hline Age (years) & $62(49-72)$ \\
No (\%) female & $80(52 \%)$ \\
No (\%) referred directly from & $59(39 \%)$ \\
external hospitals & $15(10-20)$ \\
APACHE II (ICU) & $17 \%(10-29)$ \\
APACHE II risk of death & $26(12-48)$ \\
ICU length of stay (days) & $19(6-41)$ \\
LFU length of stay (days) & \\
\hline \multicolumn{2}{l}{ All values are median (interquartile range) unless stated } \\
otherwise.
\end{tabular}

dystrophy, and eight others). Only two had neuropathy/ myopathy acquired in the ICU.

Figure 1 shows the overall outcomes for the whole group. $53 \%$ of the 111 survivors were fully weaned from ventilation. Of the $27 \%(n=42)$ who died before leaving hospital, two patients subsequently deteriorated and died despite initially weaning from ventilation. Re-institution of invasive support was considered inappropriate in both cases. There were 56 patients referred who had spent less than 21 days ventilated on the referring ICU. Mortality was lower in these patients $(14 \%(8 / 56) v 34 \%(35 / 97) ; \mathrm{p}=0.01)$. However, there was no difference in eventual rates of ventilator independence $(45 \%$ $(25 / 56) v 34 \%(33 / 97) ; \mathrm{p}=0.26)$.

Of the survivors, $71 \%(79 / 111)$ were discharged directly home from the LFU and 9\% (10/111) were transferred to other medical wards in the hospital before discharge home. Twenty eight patients were transferred to outside hospitals, six of whom died.

Fourteen patients $(9 \%)$ had been receiving ventilatory support before admission to the ICU ( six tracheostomy, eight NIV). Two of these patients had central hypoventilation due to cerebrovascular accidents, one had obesity hypoventilation, and the remainder had neuromuscular/chest wall conditions. One patient died and the 13 survivors continued to require ventilatory support at discharge.

The majority of patients $(78 \%, \mathrm{n}=120)$ were ventilated via a tracheostomy on admission, but only $16 \%$ of survivors $(\mathrm{n}=18)$ had a tracheostomy at discharge. Mini-tracheostomies to aid suctioning of secretions were used in a small number of patients who weaned from ventilation. In five out of six patients this was later removed.

Table 2 shows the outcomes related to the diagnostic groups. Patients with neuromuscular and chest wall disorders had the lowest hospital mortality (15\%) but also the lowest rate of weaning from ventilation $(19 \%)$. The mortality of the COPD group (24\%) was intermediate between the other two large diagnostic groups and was similar to the group as a whole $(27 \%)$. Over half the patients with COPD $(56 \%)$ were weaned from mechanical ventilation. Although surgical patients had the highest short term mortality (50\%), most of the survivors were weaned from ventilation (72\%, 13/ 18 survivors).

\section{Factors associated with mortality and independence from mechanical ventilation}

Univariate analysis is shown in table 3. Age, duration of stay in the referring ICU, APACHE II risk of death, and a surgical cause for admission were associated with increasing mortality. Neuromuscular/chest wall conditions were associated with low mortality but also with a low likelihood of ventilator independence. Interestingly, a diagnosis of COPD was associated with an increased likelihood of weaning from mechanical ventilation. Female sex was associated with weaning from mechanical ventilation. There was a trend towards increasing ICU length of stay being related to a reduced rate of ventilator independence $(\mathrm{p}=0.07)$. Referral from the "in house" ICU at St Thomas' Hospital was associated with an increased likelihood of weaning from ventilation. However, this group included 14 patients originally from external hospitals. $39 \%$ of patients $(n=59)$ were directly referred to the LFU from outside ICUs. Duration of stay in the referring ICU was higher among patients referred from external sources (median 41 days (IQR 25-67) $v 21$ days (IQR 9-35); $\mathrm{p}<0.001$ ). There were more patients with neuromuscular/chest wall conditions referred from outside hospitals $(27 / 59 v 20 / 94, \mathrm{p}=0.003)$. There were no other significant differences between patients from different referral sources. 


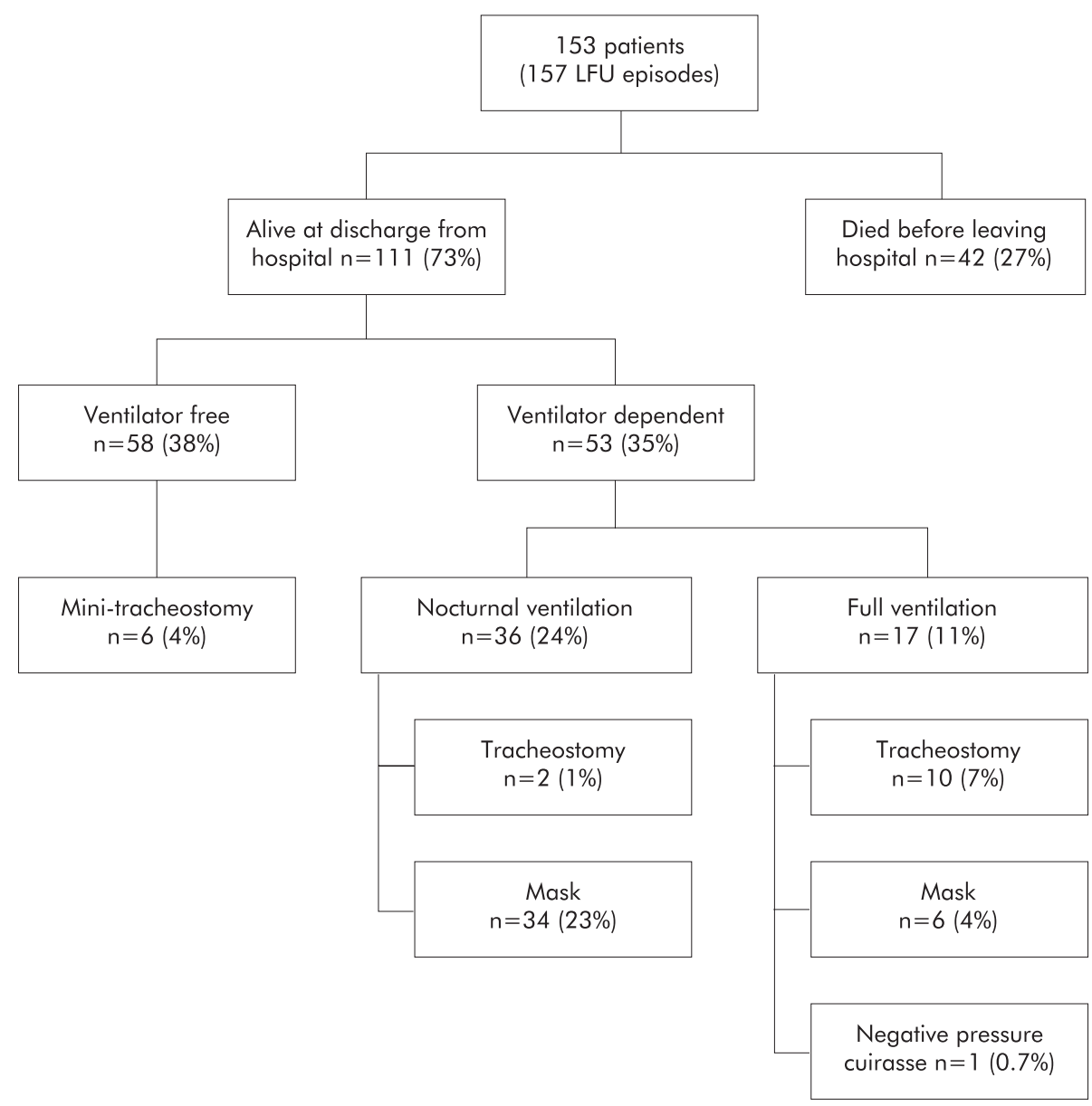

Figure 1 Survival and weaning outcomes. All percentages are expressed with 153 patients as the denominator. The mean time to ventilator independence in patients who weaned was 17 days (IQR 12-35).

\section{Cost analysis}

There was a progressive (but not statistically significant) increase in the daily charge each year $(o=0.08, \mathrm{p}=0.4$; table 4).

\section{Long term survival}

Data on 140 patients were available to assess long term survival (fig 2); $58 \%$ were alive at 1 year and $47 \%$ at 3 years. Of the 13 patients who were lost to follow up, four had moved abroad and nine were no longer traceable after discharge.

Figure 3 shows the long term survival for patients in the three main diagnostic groups who left hospital alive. The surgical and neuromuscular/chest wall patients had similar long term survival rates. Poorest survival was seen in the COPD group. After adjustment for age and time spent ventilated in the referring ICU, the differences in survival between these three groups were no longer significant.

\section{DISCUSSION}

Our study is the first from the UK to examine the outcome in weaning patients from prolonged mechanical ventilation with attention to economic factors, diagnostic issues, and long term survival.

We found an overall mortality rate of $27 \%$ and full ventilator independence of $38 \%$. Other centres have reported mortality of $7-67 \%$ with rates of ventilator independence of $25-75 \% .{ }^{1-12}$ This reflects not only operational differences between units but also differences in selection criteria, variation in expertise, and differences in patient characteristics. Units with restrictive acceptance criteria have reported higher rates of success in achieving ventilator independence and better survival. ${ }^{126}$ Referrals to the LFU were at the discretion of regional hospitals. Acceptance of patients was determined by resource availability (bed and nursing staff) rather than on the basis of perceived ability to wean.

Long term survival after admission to a specialised weaning centre has been reported to be between $15 \%{ }^{12}$ and

Table 2 Outcome related to diagnostic group

\begin{tabular}{llll}
\hline Diagnostic group & Ventilator free & Ventilator dependent & Dead \\
\hline Neuromuscular/chest wall $(n=47)$ & $19 \%(n=9)$ & $66 \%(n=31)$ & $15 \%(n=7)$ \\
COPD $(n=41)$ & $56 \%(n=23)$ & $20 \%(n=8)$ & $24 \%(n=10)$ \\
Surgical $(n=36)$ & $36 \%(n=13)$ & $14 \%(n=5)$ & $50 \%(n=18)$ \\
Other diagnoses $(n=29)$ & $41 \%(n=12)$ & $35 \%(n=10)$ & $24 \%(n=7)$ \\
\hline
\end{tabular}

Percentages are expressed as a proportion of the total number in each diagnostic group. 
Table 3 Univariate analysis of factors associated with mortality and independence from mechanical ventilation

\begin{tabular}{|c|c|c|c|c|}
\hline & \multicolumn{2}{|l|}{ Mortality } & \multicolumn{2}{|c|}{ Ventilator independence } \\
\hline & OR $(95 \% \mathrm{Cl})$ & p value & OR $(95 \% \mathrm{Cl})$ & p value \\
\hline Age (years) & $1.06(1.03$ to 1.1$)$ & $<0.001$ & $0.99(0.97$ to 1.01$)$ & 0.38 \\
\hline Female sex & 1.01 (0.49 to 2.05$)$ & 0.99 & 2.13 (1.09 to 4.17$)$ & 0.03 \\
\hline Referral from "in house" ICU & $0.99(0.54$ to 2.31$)$ & 0.760 & $5.10(2.32$ to 11.24$)$ & $<0.001$ \\
\hline Length of stay in ICU (days) & $1.02(1.01$ to 1.03$)$ & 0.001 & $0.99(0.98$ to 1.00$)$ & 0.07 \\
\hline Length of stay in LFU (days) & 1.00 (0.99 to 1.01$)$ & 0.55 & $0.99(0.99$ to 1.00$)$ & 0.15 \\
\hline APACHE II score & $1.07(0.98$ to 1.16$)$ & 0.11 & $0.99(0.91$ to 1.07$)$ & 0.73 \\
\hline APACHE II risk of death (\%) & $1.02(1.0$ to 1.05$)$ & 0.05 & 0.99 (0.96 to 1.01$)$ & 0.34 \\
\hline \multicolumn{5}{|l|}{ Underlying diagnosis } \\
\hline Neuromuscular/chest wall & $0.36(0.14$ to 0.87$)$ & & $0.28(0.12$ to 0.63$)$ & \\
\hline COPD & $1.24(0.35$ to 1.84$)$ & 0.007 & 2.81 (1.35 to 5.86$)$ & 0.015 \\
\hline Surgical & $4.04(1.79$ to 9.10$)$ & & $1.29(0.57$ to 2.90$)$ & \\
\hline
\end{tabular}

ICU, intensive care unit; LFU, Lane Fox Respiratory Unit; COPD, chronic obstructive pulmonary disease.

Odds ratios (ORs) for continuous variables represent increased risk associated with 1 unit increase in the variable. Odds ratios (ORs) for diagnostic categories are referenced against those without the condition.

$\mathrm{p}$ value for diagnostic categories is for heterogeneity across groups.

$76 \%{ }^{3}$ at 1 year and as good as $54 \%$ at 4 years. ${ }^{1}$ We found overall survival rates of $58 \%$ at 1 year and of $47 \%$ at 3 years. To our knowledge, only Schonhofer et al $l^{5}$ have examined long term survival in separate subpopulations referred to a weaning centre.

Although $76 \%$ of our patients with COPD survived to hospital discharge, only $43 \%$ of the survivors were alive at 3 years, similar to that reported by Schonhofer et al. ${ }^{5}$ COPD has been reported as the cause of ventilator dependence in approximately $25 \%$ of all patients who remain ventilator dependent 3 weeks after ICU admission. ${ }^{14}$ Specialised weaning centres report rates of liberation from ventilation in COPD of between $22 \%{ }^{7}$ and $35 \% .{ }^{4}$ However, in our study, COPD was not associated with a failure to wean-in fact, quite the reverse. Over half of this group (56\%) were liberated from ventilation. Admission to ICU in the UK has traditionally been restrictive for patients with COPD. Without measurement of pre-morbid lung function, which was rarely available, it is not possible to determine if the relatively high success of weaning we report was because they were a group who had less severe airflow limitation. Our intensive use of NIV as a bridge to spontaneous breathing may explain the difference. This has proven efficacy in treating acute exacerbations of $\mathrm{COPD}^{15-17}$ and in facilitating extubation in the ICU. ${ }^{18}{ }^{19}$ Our data suggest that many patients with COPD who have failed to wean in the ICU can subsequently be liberated from ventilation in centres with appropriate experience and facilities. However, the long term mortality for these patients remains significant.

The patients with neuromuscular/chest wall conditions were a mixed group with both stable (for example, limb girdle myopathy) and progressive diseases (for example, motor neurone disease). Despite a low initial mortality and satisfactory long term survival, a large proportion required persistent ventilatory assistance. This was predominantly nocturnal ventilation $(n=20 / 31)$ with independence for much of the day. Smith et al, ${ }^{3}$ whose patients had mainly neuromuscular diseases, reported similar findings with a low mortality $(10 \%)$ and high need for continuing ventilatory support (75\%). Schonhofer et al also found better long term survival in patients with neuromuscular conditions than in those with COPD. In our study a high proportion of patients with neuromuscular/chest wall conditions were referred from outside ICUs, which may explain the lower rate of ventilator independence in those referred from external sources. The low mortality in this group suggests that referral to a weaning centre should occur when weaning is delayed, especially as it is likely that there will be a need for continued domiciliary ventilatory support.

Half of the surgical group died before leaving hospital. However, the majority of survivors $(69 \%, 11 / 16)$ were still alive at 3 years. Reports that have analysed outcome related to delayed weaning in surgical patients give higher rates of survival $^{10}$ and ventilator independence ${ }^{7}$ than in medical patients. The older age (71 years (IQR 60-75) $v 61$ years (IQR 47-68); $\mathrm{p}=0.03$ ) in our surgical patients compared with the rest of the group may have been a factor in the higher initial mortality. There was insufficient information to assess fully the effect of coincident medical conditions such as COPD and cardiovascular disease which may have contributed to the high initial mortality observed.

Factors associated with increasing hospital mortality were age, duration of stay in the referring ICU, and APACHE II risk of death. Both Swinburn et $a l^{20}$ and Cohen $e t a l^{21}$ report poor outcomes in elderly ventilator dependent patients. Despite elderly patients having a higher mortality, age per se did not affect the likelihood of weaning from prolonged mechanical ventilation. The association of ICU length of stay and

Table 4 Cost analysis from 1997 to 2000

\begin{tabular}{lll}
\hline Year & Cost per LFU stay $(€)$ & Cost per day $(€)$ \\
\hline 1997 & $29150(13800-49600)$ & $1290(1190-2420)$ \\
1998 & $36500(15950-66850)$ & $1340(1230-2550)$ \\
1999 & $28450(23590-58150)$ & $1350(1290-1490)$ \\
2000 & $38150(21350-81600)$ & $1470(1280-1940)$ \\
$1997-2000$ & $33050(16050-70600)$ & $1350(1250-2040)$ \\
\hline LFU, Lane Fox Respiratory Unit. & \\
Values are shown as medians (IQR). \\
Costing was originally evaluated in pounds sterling and has been converted to Euros using £l=€ 1.5026.
\end{tabular}




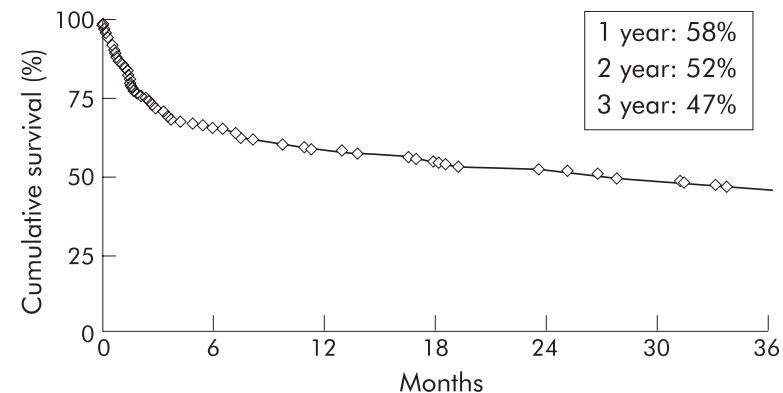

Figure 2 Long term survival in 140 patients.

mortality has been previously reported ${ }^{22}$ and is likely to be related to both the underlying diagnosis and complications such as nosocomial infection.

The APACHE II predicted risk of death, which was recorded on admission to the referring ICU, predicted mortality even after admission to the LFU (OR 1.024, $\mathrm{p}=0.046)$. The relationship between severity scoring systems, ${ }^{23}{ }^{24}$ physiological parameters, ${ }^{41}{ }^{25}$ and outcome has previously been investigated. There is, however, little published work incorporating both physiological measurements and the underlying diagnosis to help predict outcome. Another factor associated with successful weaning was female sex. Why this should be a factor is unclear, but Scheinhorn $\mathrm{et} \mathrm{al}^{23}$ report similar findings. Studies designed to look specifically at the effect of sex on ICU outcomes have not found any association with mortality or length of stay in the ICU. ${ }^{26}$

We know of no published data from the UK to allow comparison of the costs involved in caring for weaning failure patients. Using a similar method of assessment it has been estimated that the cost of stay in an ICU is almost double the cost in a weaning centre. ${ }^{27}$ These reduced costs principally arise from the lower staffing ratio. Centres in the USA have shown financial savings compared with ICU care. ${ }^{28} 29$ Given the pressure on ICU facilities and the effect on activityespecially in smaller units - through blocking of an ICU bed by long stay patients, there is a strong case for the expansion or creation of regional weaning units such as ours.

\section{Limitations of the study}

Our study is a retrospective review of all patients referred and transferred to a single unit over a 4 year period. The data collection and analysis were done independently of the clinicians caring for the patients. The number of patients is smaller than from longer established weaning centres in the USA. ${ }^{1}$ 4 $^{10}$ With the exception of data from Papworth, ${ }^{3}$ nothing has previously been published from the UK. $37 \%$ of our patients did not meet the generally accepted definition for weaning failure (ventilated $\geqslant 21$ days), but all were referred because of anticipated weaning difficulty. Over half the patients were referred from the ICU at St Thomas' Hospital. The reduced length of ICU stay in these patients may have been due either to ease in transferring in-house patients or to a delay in referral of external patients. The statistical analysis shows only the univariate associations. Despite this, the underlying diagnosis remains important and clinically relevant in determining early and late outcomes.

\section{Conclusions}

We conclude that many survivors of prolonged mechanical ventilation can be weaned from ventilatory support. Younger patients and those who spent less time in the referring ICU were most likely to survive. Patients with neuromuscular/ chest wall conditions had good long term survival but few

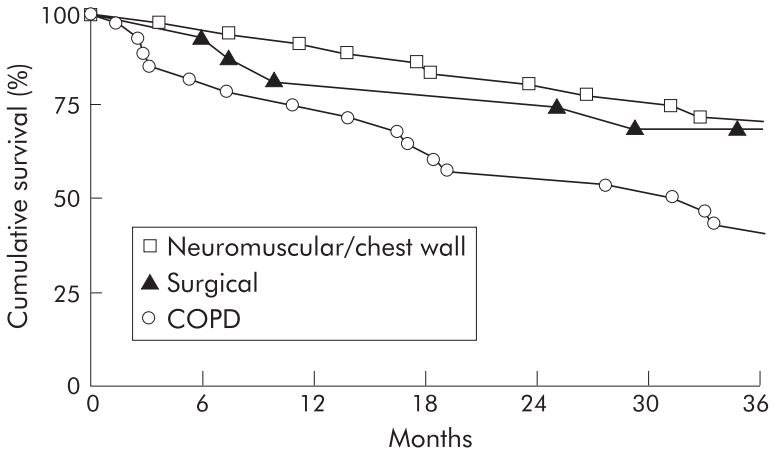

\begin{tabular}{lcc}
\hline & 1 year & 3 years \\
\hline Neuromuscular/chest wall $(n=36)$ & $89 \%$ & $70 \%$ \\
Surgical $(n=16)$ & $81 \%$ & $69 \%$ \\
COPD $(n=28)$ & $75 \%$ & $43 \%$ \\
\hline
\end{tabular}

Figure 3 Long term survival in patients who left hospital alive according to diagnostic group.

were totally liberated from ventilatory support. Long term survival in COPD was poor but, for many, weaning proved possible. Patients with postoperative weaning failure had a high initial mortality but survival after hospital discharge was good. The underlying diagnosis was crucial in determining early and long term survival and the likelihood of weaning from mechanical ventilation.

\section{Authors' affiliations}

D V Pilcher, D F Treacher, A J Williams, A C Davidson, Lane Fox Respiratory Unit, Guy's and St Thomas' Hospital, London SEl 7EH, UK M J Bailey, Department of Epidemiology and Preventive Medicine, Monash University, Melbourne, Australia

S Hamid, Bromley Hospitals NHS Trust, Bromley, Kent BR6 8ND, UK

\section{REFERENCES}

1 Gracey DR, Hardy DC, Naessens JM, et al. The Mayo Ventilator-Dependent Rehabilitation Unit: a 5 year experience. Mayo Clin Proc 1997;72:13-9.

2 Gracey DR, Naessens JM, Viggiano RW, et al. Outcome of patients cared for in a ventilator-dependent unit in a general hospital. Chest 1995; 107:494-9.

3 Smith IE, Shneerson JM. A progressive care programme for prolonged ventilatory failure: analysis of outcome. Br J Anaesth 1995;75:399-404.

4 Dasgupta A, Rice R, Mascha E, et al. Four-year experience with a unit for long-term ventilation (respiratory special care unit) at the Cleveland Clinic Foundation. Chest 1999;116:447-55.

5 Schonhofer B, Euteneuer S, Nava S, et al. Survival of mechanically ventilated patients admitted to a specialised weaning centre. Intensive Care Med 2002;28:908-16.

6 Scheinhorn DJ, Chao DC, Stearn-Hassenpflug MS, et al. Post-ICU mechanical ventilation. Treatment of 1123 patients at a regional weaning centre. Chest 1997;111:1654-9.

7 Bagley PH, Cooney E. A community-based regional ventilator weaning unit. Chest 1997;111:1024-9.

8 Indihar FJ. A 10-year report of patients in a prolonged respiratory care unit. Minn Med 1991;74:23-7

9 Douglas SL, Daly BJ, Brennan PF, et al. Outcomes of long-term ventilator patients: a descriptive study. Am J Crit Care 1997;6:99-105.

10 Latriano B, McCauley P, Astiz ME, et al. Non-ICU care of hemodynamically stable mechanically ventilated patients. Chest 1996;109:1591-6.

11 Carson SS, Bach PB, Brzozowski L, et al. Outcomes after long-term acute care. An analysis of 133 mechanically ventilated patients. Am J Respir Crit Care Med 1999:159:1568-73.

12 Elpern EH, Larson R, Douglass P, et al. Long-term outcomes for elderly survivors of prolonged ventilator assistance. Chest 1989;96:1120-4.

13 Dickie H, Vedio A, Dundas R, et al. Relationship between TISS and ICU costs. Intensive Care Med 1998;24:1009-17.

14 Hamid S, Noonan YM, Williams AJ, et al. An audit of weaning from mechanical ventilation in a UK centre. Thorax 1999;54:86P.

15 Brochard L, Mancebo J, Wysocki $M$, et al. Noninvasive ventilation for acute exacerbations of chronic obstructive pulmonary disease. N Engl J Med 1995:333:817-22.

16 Plant PK, Owen JL, Elliott MW. Early use of non-invasive ventilation for acute exacerbations of chronic obstructive pulmonary disease on general respiratory wards: a multicentre randomised controlled trial. Lancet 2000;355:1931-5. 
17 Bott J, Carroll MP, Conway JH, et al. Randomised controlled trial of nasal ventilation in acute ventilatory failure due to chronic obstructive airways disease. Lancet 1993;341:1555-7.

18 Nava S, Ambrosino N, Clini E, et al. Noninvasive mechanical ventilation in the weaning of patients with respiratory failure due to chronic obstructive pulmonary disease. A randomized, controlled trial. Ann Intern Med 1998; 128:721-8.

19 Hilbert G, Gruson D, Portel L, et al. Noninvasive pressure support ventilation in COPD patients with postextubation hypercapnic respiratory insufficiency. Eur Respir J 1998:11:1349-53.

20 Swinburne AJ, Fedullo AJ, Bixby K, et al. Respiratory failure in the elderly patient in intensive care: analysis of outcome after treatment with mechanical ventilation. Arch Intern Med 1993;153:1657-62.

21 Cohen IL, Lambrianos J, Fein Al. Mechanical ventilation for the elderly patien in the intensive care: incremental charges and benefits. JAMA 1993;269:1025-9

22 Mahul TR, Perrot D, Temelhoff G. Short- and long-term prognosis, functional outcome following ICU for the elderly. Intensive Care Med 1991;17:7-10.
23 Scheinhorn DJ, Stearn-Hassenpflug MS, et al. Predictors of weaning after 6 weeks of mechanical ventilation. Chest 1995; 107:500-5.

24 Gluck EH, Corigan L. Predicting Eventual success or failure to wean in patients receiving long-term ventilation. Chest 1996;1 10:1018-24.

25 Chao DC, Scheinhorn DJ, Stearn-Hassenpflug MS. Impact of renal dysfunction on weaning from prolonged mechanical ventilation. Crit Care (Lond) 1997; 1:101-4.

26 Epstein SK, Van Vuong BS. Lack of influence of gender on outcomes of mechanically ventilated medical ICU patients. Chest 1999;1 16:732-9.

27 Baudouin SV, Davidson AC, Elliott MW, et al. National Patients Access Team Critical Care Programme: weaning and long term ventilation. London: Department of Health, 2002 (available at, http://www.modern.nhs.uk/ critical care)

28 Gracey DR, Hardy DC, Koenig GE. The chronic ventilator dependent unit: lower cost alternative to intensive care. Mayo Clin Proc 2000;75:445-9.

29 Seneff MG, Wagner D, Thompson D, et al. The impact of long-term acute-care facilities on the outcome and cost of care for patients undergoing prolonged mechanical ventilation. Crit Care Med 2000;28:342-50.

\section{LUNG ALERT}

An increased risk of community acquired pneumonia with gastric acid suppressive drug therapy

$\Delta$ Laheij RJF, Sturkenboom MCJM, Hassing RJ, et al. Risk of community-acquired pneumonia and use of gastric acidsuppressive drugs. JAMA 2004;292:1955-60

7

his study analysed 364683 patients from an established Dutch primary care database comprising full electronic patient records. Patients were described as exposed $(\mathrm{n}=19459)$ if they were prescribed acid suppression (proton pump inhibitor (PPI) or $\mathrm{H}_{2}$ receptor antagonist $\left.\left(\mathrm{H}_{2} \mathrm{RA}\right)\right)$ and were followed until the development of pneumonia or the end of the study period. A total of 977893 patient-years of data were obtained. The unadjusted relative risk of pneumonia in the exposed group was 4.5 (95\% CI 3.8 to 5.1 ) compared with the non-exposed group. A nested case-control study using 475 patients and 4690 controls was performed to attempt to allow for confounding by indication. Adjusted relative risks for pneumonia in those currently using PPIs or $\mathrm{H}_{2} \mathrm{RAs}$ were 1.89 (95\% CI 1.36 to 2.62 ) and 1.63 (95\% CI 1.07 to 2.48 ), respectively. Interestingly, there appeared to be a dose-response relationship for those taking PPIs and their risk of pneumonia that was not apparent for $\mathrm{H}_{2}$ RAs. The conclusion is that gastric acid suppression is associated with an increased risk of community acquired pneumonia.

The concept of gastric acid suppression and the development of pneumonia is longstanding and has biological plausibility. Evaluating the link using a randomised controlled trial is unlikely to be feasible. This study has flaws which the authors recognise: its observational nature, the definition of pneumonia, and that confounding variables are possible even with the nested case-control study. This paper will not alter prescribing habits as gastric acid suppression is effective and has an enviable safety record. It should remind us that few treatments are without side effects and that care is needed in high risk patients (elderly, those with pre-existing lung disease, and immunosuppression).

R Parker Specialist Registrar in Respiratory Medicine, Oxford Centre for Respiratory Medicine, Churchill Hospital, Oxford, UK; robertparker@doctors.org.uk 